\title{
Preparation and Rheology of Magnetorheological Fluid Using Six Kinds of Fumed Silica as Stabilizing Additives
}

\author{
Makoto KANDA $^{1}$, Aya KAIDE ${ }^{2}$, Takashi SAEKI ${ }^{2 *}$, and Hiroshi TOCHIGI ${ }^{1}$ \\ ${ }^{1}$ Product Research Institute, Cosmo Oil Lubricants Co., Ltd., 1134-2, Gongendo, Satte-shi, Saitama 340-0193, Japan \\ ${ }^{2}$ Graduate School of Science and Technology for Innovation, Yamaguchi University, 2-16-1 Tokiwadai, Ube-shi, Yamaguchi 755- \\ 8611, Japan
}

\begin{abstract}
In this study, magnetorheological fluid (MRF) was prepared with several kinds of hydrophobic fumed silica in order to improve MRF stability while maintaining desirable fluidity. We conducted steady flow measurements and oil separation tests under gravity and centrifugal conditions. The linkage of fumed silica particles was also observed morphologically by transmission electron microscopy. We found that the MRF sedimentation stability correlated with viscosity in the lower shear rate range. The oil separation ratio improved with the addition of the fumed silicas examined in this study. The surface characteristics of the silica particles affected the stabilization ability. The addition of hydrophobic fumed silica can desirably increase MRF magnetic properties.
\end{abstract}

\section{Introduction}

Magnetorheological fluid (MRF) is a slurry prepared with oil and magnetic particles that have an average diameter of several micrometers. MRF is classified as a functional fluid since its viscosity can be reversibly controlled by a magnetic field (Rabinow, 1948). The quick and large variations in the rheological property of MRF with respect to a magnetic field (Weiss et al., 1994; Carlson, 1994) are desirable for applications such as automotive dampers, brakes, and clutches (Kordonsky, 1993; Carlson, 1996; Jolly and Nakano, 1998; Nakano et al., 2009). For MRF to have good fluidity under in the absence of a magnetic field, a suitable dispersant is generally added to prevent viscosity increase due to particle aggregation. Moreover, the difference in density between the oil and the particles in MRF is large, resulting in particle sedimentation and liquid separation of the upper layer. Such unstable slurries simultaneously cause unstable magnetic properties of MRF, leading to various problems such as device malfunction and damage due to the occlusion and deposition of magnetic particles. Therefore, the development of a technique to increase MRF stability is necessary. In the case of MRFs used for automotive dampers, the desired viscosity range is 0.01 to $1 \mathrm{~Pa} \cdot \mathrm{s}$ (at $40^{\circ} \mathrm{C}$ ) (Weiss et al., 1994). The use of high-viscosity oil as a solvent showed a stabilizing effect on MRF; however, it is not desirable to deviate from this range. Also, higher-viscosity MRF is not attractive for use in rotating devices such as brakes and clutches from the viewpoint of energy saving, because a high shear rate is applied in the drive part of the device.

Lim et al. (2004) reported a method to reduce the diameters of magnetic particles to a sub-micron level in order to prevent sedimentation. However, the stress intensity when a magnetic field is applied depends on the particle size. MRF with small magnetic particles cannot be used for large machines and vehicles. Song et al. (2009) and Park et al. (2009) reported that the addition of $1 \mathrm{wt} \%$ nanoparticles was effective for improving MRF stability and the MR effect. Lim et al. (2004) prepared MRF using fumed silica to prevent oil separation. Since they did not use any dispersants, their MRF had a higher viscosity $\left(2 \mathrm{~Pa} \cdot \mathrm{s}\right.$ or more at a shear rate of $\left.100 \mathrm{~s}^{-1}\right)$ than practical MRF products. Fumed silica is commonly used as a paint additive whose nanoparticles are expected to agglomerate and/or coagulate and thus to increase the stabilization of slurries. When we use fumed silica as an MRF stabilizer, the effect of the presence of a dispersant as well as the surface characteristics of fumed silica are important factors. In this study, several kinds of hydrophobic fumed silica and a hydrophilic one were examined as MRF stabilizers. These silicas were screened to identify the one that most effectively increased MRF stability while maintaining good fluidity. The rheological properties of each fumed silica dispersed in a base oil were measured. Transmission electron microscopy (TEM) was used to observe the linkage of fumed silica particles. The objective of this study was to obtain valuable information in order to clarify the

Corresponding author: makoto_kanda@cosmo-oil.co.jp 
mechanism underlying stabilization caused by such fumed silica additives.

\section{Experimental}

\subsection{Materials and preparation}

A highly refined paraffinic mineral oil (kinematic viscosity at $40^{\circ} \mathrm{C}: 2.0 \times 10^{-5} \mathrm{~m}^{2} / \mathrm{s}$, viscosity index: 122 ) was used as the solvent oil. The magnetic particles were a carbonyl iron powder (CS, BASF) with a density of $7860 \mathrm{~kg} / \mathrm{m}^{3}$. The 10,50 , and $90 \%$ pass diameters of the powder were $2.8,5.6$, and $12.3 \mu \mathrm{m}$, respectively. Oleic acid was used as a dispersant. Six kinds of fumed silica (Silica-A-F) as shown in Table 1 were selected as stabilizers. The degree of hydrophobicity was evaluated by gradually adding ethanol to fumed silica floating in water, and the ethanol concentration was determined when all silica particles were precipitated (indicated by the $E$ value). The silanol group ratio in the table indicates the ratio of hydrophobic silica surface to the hydrophilic surface of the silanol groups, which was determined as follows:

A fumed silica $(1 \mathrm{~g})$ and $35 \%$ aqueous ethanol solution $(100 \mathrm{~g})$ was stirred in a beaker to obtain a dispersed silica solution. Then, 35\% ethanol aqueous solution containing $0.01 \mathrm{~mol} / \mathrm{L} \mathrm{KOH}$ was dripped into the dispersion. The silanol group ratio was computed by using the volume of solution that had dripped into the dispersion up to the point where $\mathrm{pH}$ reached 7. characteristics were measured for shear rate in the range from 0.01 to $1000 \mathrm{~s}^{-1}$. The measurement temperature was set at $25 \pm 0.5^{\circ} \mathrm{C}$ with a Peltier air-bath control system. Rheological properties were measured within 24 hours after the MRFs were prepared.

\subsection{TEM observation}

The morphology of the structure of the fumed silica was observed using a TEM (JEM-140; JEOL, Tokyo, Japan) with an accelerating voltage of $100 \mathrm{kV}$. In the preprocessing, the sample was dissolved in the base oil, and the sample was attached to a support film grid. Since we did not conduct active drying, mineral oil was also placed on the TEM grid. Then, the sample was stained with osmium tetraoxide $2 \%$ aqueous solution for $4 \mathrm{~h}$ to achieve an osmium coating on the surfaces of the selfassembled structures.

\subsection{Oil separation test}

MRF samples were first agitated to form a uniform slurry. Ten milliliters of MRF was poured into a glass measuring cylinder having a volume of $10 \mathrm{ml}$. The cylinder was then allowed to stand at room temperature for 3 weeks. The height of the separated oil that formed as the upper layer was measured over time. Finally, the oil separation rate $\varphi$ was calculated as follows:

$$
\varphi=v / V \times 100
$$

Table 1. Properties of fumed silica.

\begin{tabular}{|c|c|c|c|c|c|c|c|c|}
\hline \multirow[b]{2}{*}{ MRF sample } & \multicolumn{8}{|c|}{ Fumed silica } \\
\hline & $\begin{array}{c}\text { Fumed silica } \\
\text { sample }\end{array}$ & Surface treatment & Characteristic & $\begin{array}{c}\text { Average primary } \\
\text { particle size } \\
(\mathrm{nm})\end{array}$ & $\begin{array}{c}\text { E value } \\
(\%)\end{array}$ & $\begin{array}{l}\text { Silanol } \\
\text { group } \\
\text { ratio }\end{array}$ & $\begin{array}{c}\text { Loss on ignition } \\
\text { for } 2 \text { hours at } \\
1000^{\circ} \mathrm{C} \\
(\%)\end{array}$ & $\begin{array}{c}\text { Carbon } \\
\text { content } \\
(\%)\end{array}$ \\
\hline MRF-A & Silica-A & $\mathrm{Si}-\mathrm{OH}$ & Hydrophilic & 12 & 0 & 1 & $<1$ & - \\
\hline MRF-B & Silica-B & $>\mathrm{Si}\left(\mathrm{CH}_{3}\right)_{2}$ & Hydrophobic & 12 & 15 & 0.47 & $<2$ & 1 \\
\hline $\mathrm{MRF}-\mathrm{C}$ & Silica-C & $-\mathrm{Si}\left(\mathrm{CH}_{3}\right)_{3}$ & Hydrophobic & 12 & 31 & 0.13 & $1.5 \sim 3.5$ & 2 \\
\hline MRF-D & Silica-D & $-\left\{\mathrm{Si}\left(\mathrm{CH}_{2}\right)_{2} \mathrm{O}-\right\}_{\mathrm{n}}-\mathrm{Si}\left(\mathrm{CH}_{3}\right)$ & Hydrophobic & 12 & 32 & 0.34 & $4 \sim 6$ & 5 \\
\hline MRF-E & Silica-E & $\equiv \mathrm{Si}-\mathrm{C}_{8} \mathrm{H}_{17}$ & Hydrophobic & 12 & 31 & 0.34 & $5 \sim 7$ & 6 \\
\hline MRF-F & Silica-F & $>\mathrm{Si}\left(\mathrm{CH}_{3}\right)_{2}$ & Hydrophobic & 16 & 15 & 0.19 & $<2$ & 1 \\
\hline
\end{tabular}

MRF was prepared with 75 wt $\%$ carbonyl iron powder and $23 \mathrm{wt} \%$ mineral oil, together with $0.25 \mathrm{wt} \%$ oleic acid and $1.75 \mathrm{wt} \%$ fumed silica. They were mixed using a bladeless rotation/revolution mixing apparatus (Awatori Rentaro ARV-310, Thinky, Tokyo, Japan) at $2000 \mathrm{rpm}$ for $5 \mathrm{~min}$ to obtain MRF samples (MRF-A-F).

\subsection{Rheological measurement}

The rheological properties of the samples were measured using a strain-control type cone and plate rheometer (MCR-101, Anton Paar, Graz, Austria). Steady flow where, $v$ and $V$ were the volume of the layer calculated from the measured height of the layer and the total volume of the oil in the MRF sample, respectively.

\subsection{Centrifugation test}

Twenty-five milliliters of MRF was poured into a centrifuge tube and centrifugal force was applied with a rotational speed of $1500 \mathrm{rpm}$ (acceleration: $650 \mathrm{G}$ ) by a centrifuge (H-215, Kokusan, Tokyo, Japan). The volume of oil separation in the MRF's upper layer was measured 
every 2 minutes. The oil separation rate was calculated in the same manner as in the oil separation test. The experiment was performed at room temperature.

\subsection{Measurement of magnetic properties}

The magnetic properties of MRF were measured by using a rheometer (MCR-101, Anton Paar) with a magneto-rheological device cell (MRD170/1T, Anton Paar) and TwinGap ${ }^{\mathrm{TM}}$ Geometry (TG/MRD, Anton Paar). The jig consisted of three parallel plates with a diameter and a gap of $19.95 \mathrm{~mm}$ and $0.3 \mathrm{~mm}$, respectively. The measurement was conducted within 24 hours after preparation of the MRF. MRF of $0.2 \mathrm{~mL}$ was cast, and a magnetic field was applied to it by means of the cell. The shear stress at the shear rate of $100 \mathrm{~s}^{-1}$ was measured with varying magnetic flux densities at $25^{\circ} \mathrm{C}$.

\section{Results and Discussion}

\subsection{Steady flow characteristic of fumed silica in mineral oil}

Figure 1 shows the steady state flow characteristics of $7 \mathrm{wt} \%$ fumed silica dispersions in the oil. The hydrophilic silica, Silica-A, showed a significant increase in viscosity across the whole range of shear rates. The viscosity in the lower shear rate range of 0.001 to $1 \mathrm{~s}^{-1}$, increased in the order of Silica-A, Silica-B, Silica-E, Silica-D, Silica-F, and Silica-C, which coincided with the order of the silanol group concentration from high to low. Fumed silica usually forms aggregates that link with each other in the solvent by the hydrogen bonding of the silanol groups. The results reflected that the strength of such linkages contributed to the surface characteristic (silanol group ratio) of the fumed silica. In the higher shear rate range of 100 to $1000 \mathrm{~s}^{-1}$, the viscosities of the hydrophobic fumed silicas (Silicas B-F) were almost the same, while that of Silica-A was around 10 times larger than these.

\subsection{TEM observation of fumed silica in mineral oil}

Figure 2 shows the TEM images of oil dispersions of 7 $\mathrm{wt} \%$ Silica-A, Silica-E, and Silica-F. We found large and concentrated coagulants in the image of Silica-A, and many small and dilute coagulant pieces in the image of Silica-E. Coagulants in the image of Silica-F were more connected than those of Silica-E. Since higher hydrophobic particles tend to disperse moderately in nonpolar oil, the higher $E$ values, silanol group ratios, and carbon contents of Silica-E may relate to the reduced linkage of coagulants in Silica E.
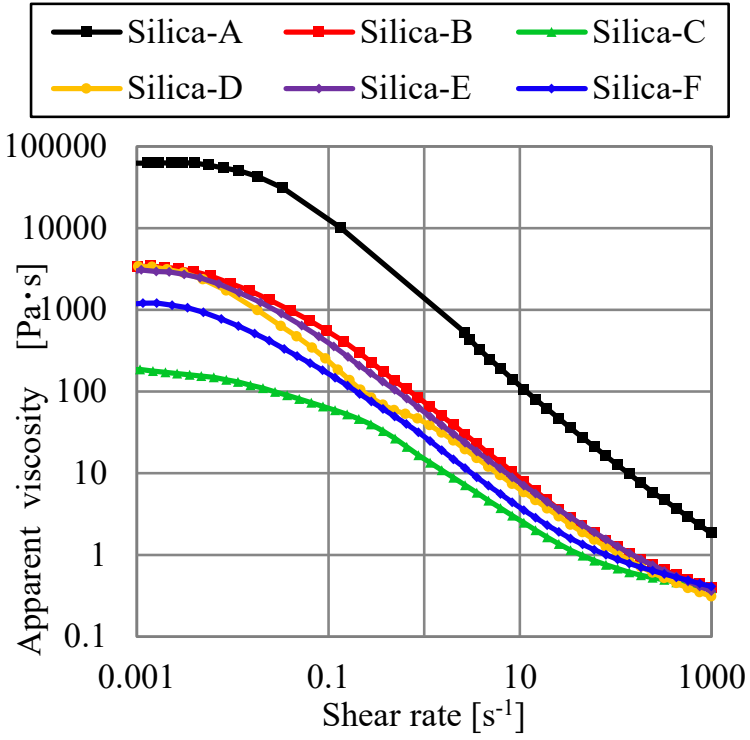

Figure 1. Steady flow characteristic of each fumed silica in mineral oil
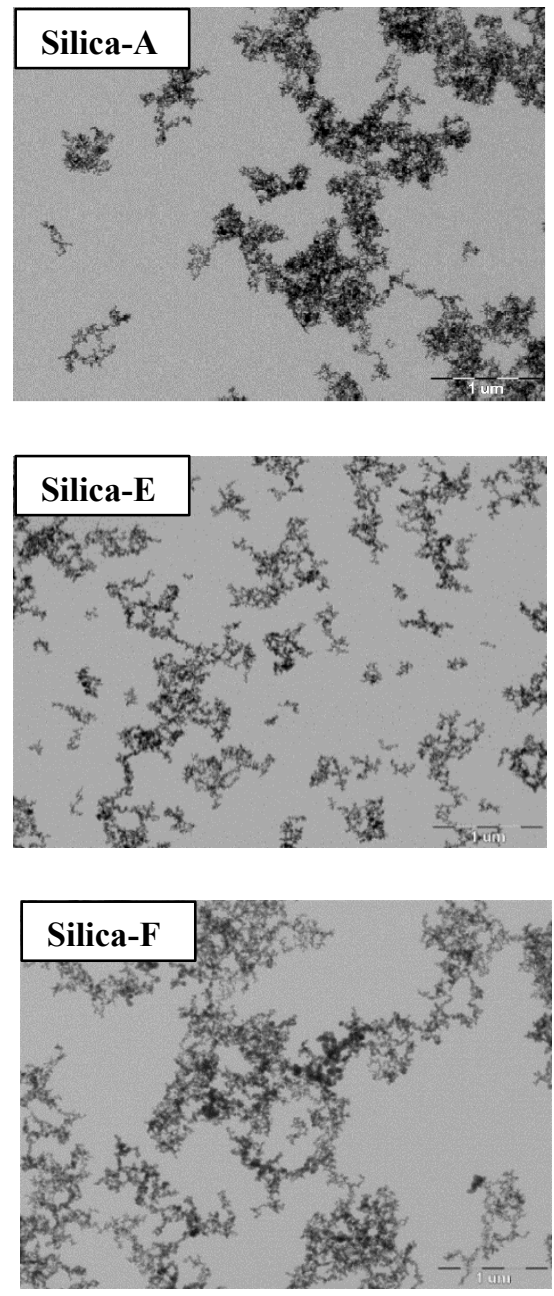

Figure 2. TEM images of fumed silica in mineral oil. 


\subsection{MRF steady flow characteristics}

Figure 3 displays the apparent viscosity at the shear rate of $10 \mathrm{~s}^{-1}$ of MRF with respect to the oleic acid concentration. We found that the addition of $0.1-0.3 \mathrm{wt} \%$ oleic acid was sufficient to reduce the apparent viscosity of MRF. This means that the addition of $1.0 \mathrm{wt} \%$ oleic acid adopted for the MRF preparation was superabundant for dispersing iron particles.

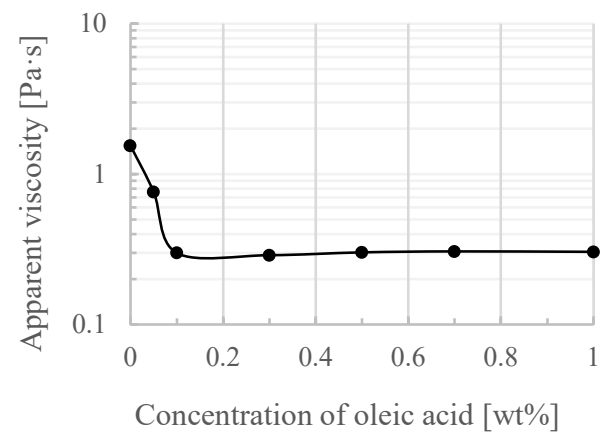

Figure 3. Apparent viscosity of MRF at different concentrations of oleic acid

Figure 4 displays the steady flow characteristics of MRFs prepared with $(\bullet)$ and without (०) oleic acid. With the addition of oleic acid, MRF viscosity significantly decreased. The added oleic acid adsorbed on the magnetic particles, which increased their dispersibility, consequently improving MRF fluidity.

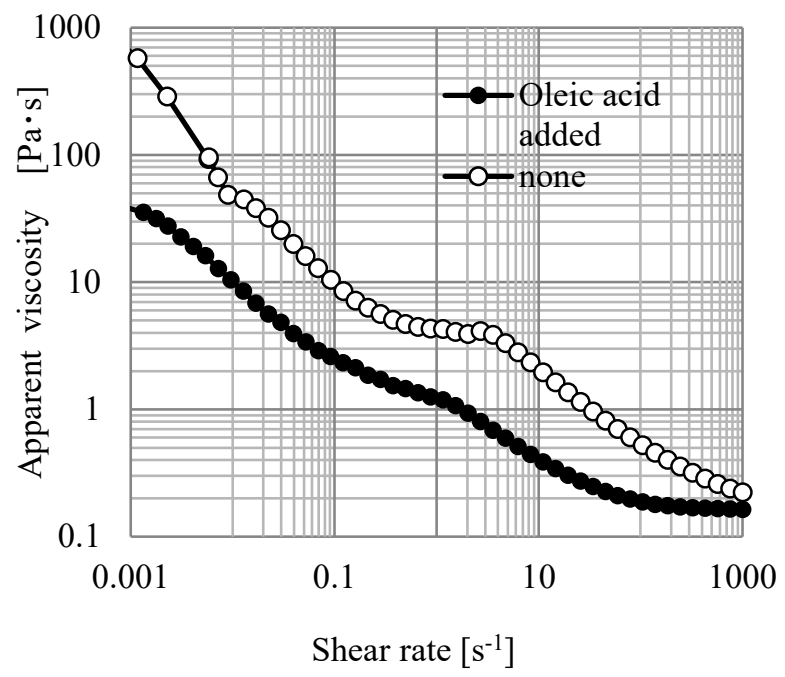

Figure 4. MRF steady flow characteristics with and without oleic acid

From the results of the oil separation test, MRF without oleic acid showed a large amount of particle sedimentation within an hour. Meanwhile, MRF with oleic acid showed remarkable and rapid sedimentation within several minutes. We expected that the aggregation structure of the magnetic particles was weakened by the presence of oleic acid.

Figure 5 shows the steady state flow characteristics of MRFs using hydrophilic (MRF-A) and hydrophobic (MRF-B) fumed silica with and without oleic acid. With the addition of oleic acid, the viscosities of both MRFs decreased, a trend similar to that shown in Figure 4. The hydrophilic silica in MRF-A without oleic acid might be coagulated, which could account for the higher viscosity of this MRF. The viscosity was significantly decreased by the dispersion effect with the addition of oleic acid. Meanwhile, MRF-B showed a slight decrease in viscosity with the addition of oleic acid. We considered that the smaller effect of oleic acid in the latter case revealed that oleic acid was less adsorbed onto the surface of hydrophobic silica. The viscosities of all four MRF samples were almost the same at the shear rate of $1000 \mathrm{~s}^{-1}$.

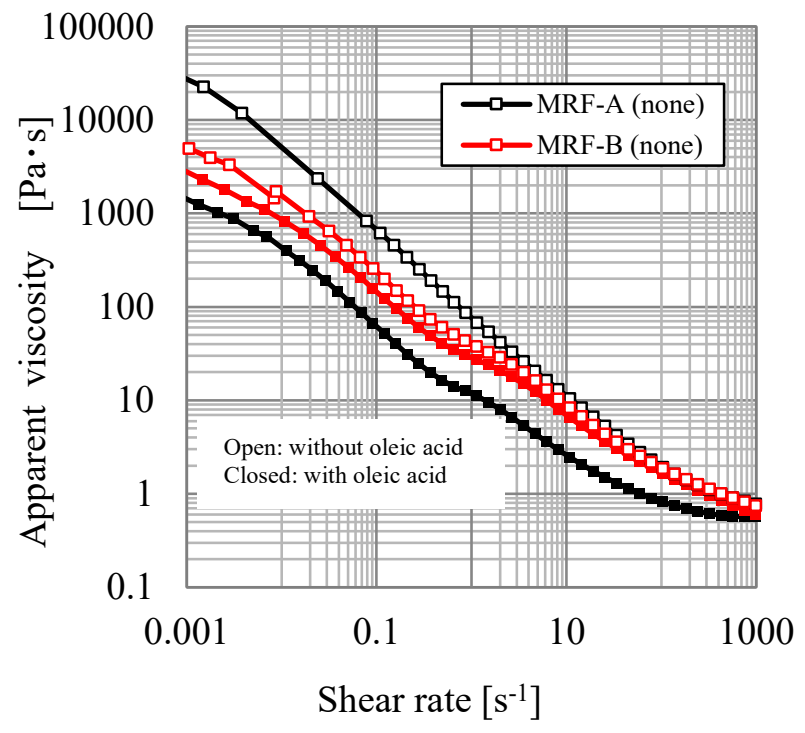

Figure 5. Steady flow characteristics of MRF-A and MRF-B with and without oleic acid

Figure 6 shows the steady state flow characteristics of MRFs prepared with the five hydrophobic fumed silicas with oleic acid. The trend and the order were similar to the results shown in Figure 1, except for MRF-E; however, we cannot explain this phenomenon in detail because the relationships among the iron particles, fumed silica particles, and oleic acid are not clear. Comparison of the results of MRF-B and MRF-F shows that the viscosity of MRF with the addition of larger particles (MRF-F) was lower.

\subsection{MRF stability}

Figure 7 shows the results of the oil separation tests. Sudden separation occurred until 50 hours for all the MRF samples. The formation of an oil layer tended to be high as the value of silanol group ratio of the silica increased, except for MRF-A (hydrophilic) and MRF-F (larger particle size); all the samples became saturated 
after 168 hours. MRF-B, which showed the highest viscosity in the lower shear rate range in Figure 5, displayed the lowest oil separation ratio. This might be related to the fact that the internal structure built by the stabilizer correlates with MRF stability (Kanda et al., 2019).

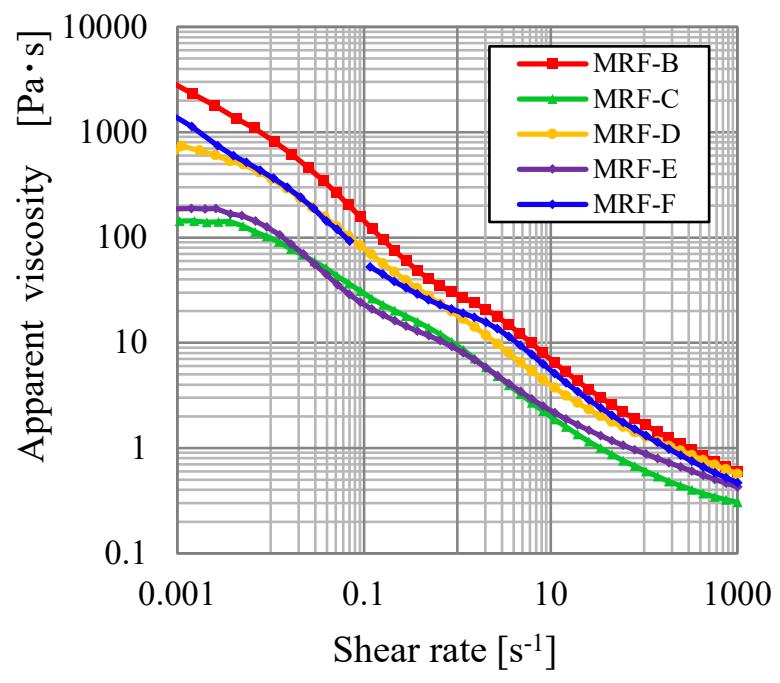

Figure 6. Steady flow characteristics of MRFs with hydrophobic fumed silica

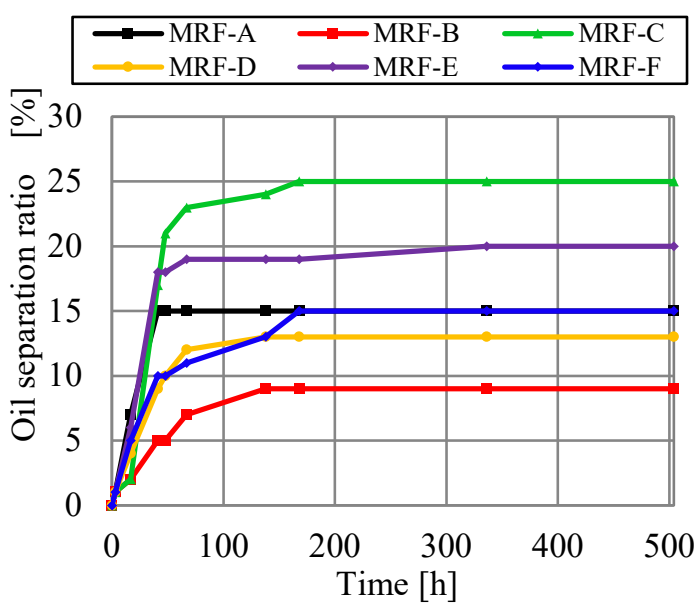

Figure 7. Oil separation ratio of MRF (oil separation test)

Figure 8 shows the oil separation results obtained from the centrifugation tests. Although the applied centrifugal acceleration was high $\left(6,370 \mathrm{~m} / \mathrm{s}^{2}\right)$, both MRF-B and MRF-F showed only $12 \%$ oil separation after $2 \mathrm{~min}$. The different results of the oil separation test for MRF-F (indicating a higher ratio than MRF-B) and the centrifugal test (coinciding with MRF-B) suggested that acceleration was not the only factor in the differing results between MRF-B and MRF-F.
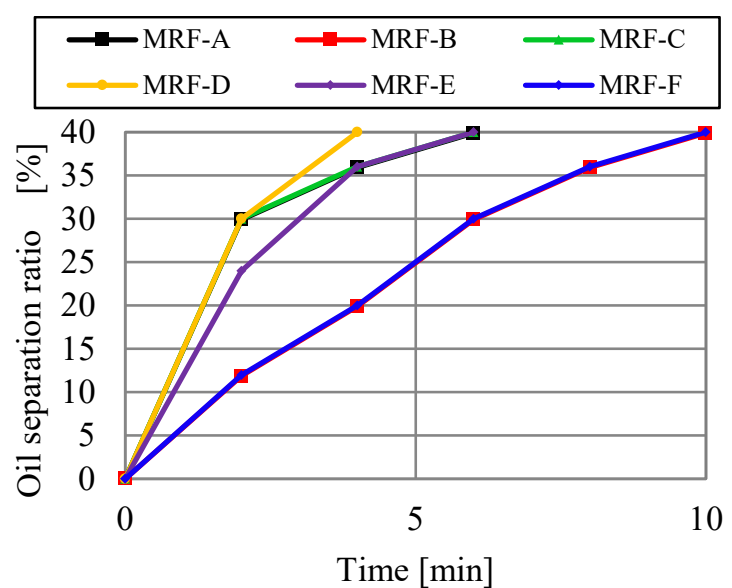

Figure 8. MRF oil separation ratio (centrifugation test)

\subsection{MRF magnetic properties}

Figure 9 shows the magnetic properties of MRFs. All the MRFs with hydrophobic fumed silica (MRF-B-F) showed higher stress compared to MRF-A with hydrophilic fumed silica. In other words, the addition of hydrophobic fumed silica was effective for improving the magnetic properties of MRF with oleic acid. Each fumed silica can function to augment the linkage between iron particles. Consequently, the thus-obtained structure may be subject to greater shear stress under a magnetic field. No significant difference was observed among the MRFs with hydrophobic fumed silica.

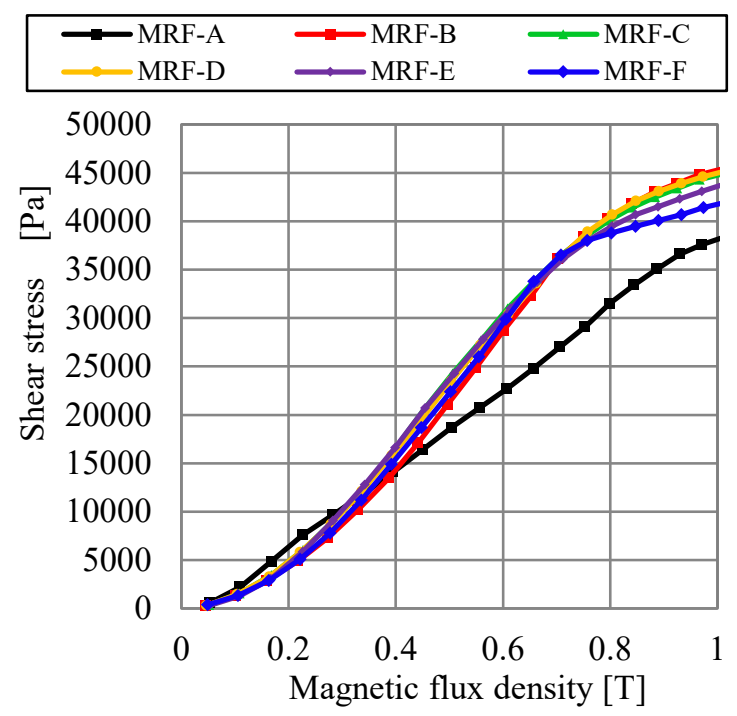

Figure 9. Magnetic characteristics of MRFs with fumed silica

\section{Conclusion}

Fumed silica additives having different surface characteristics were used as MRF stabilizers. With regard to fluidity, it is convenient to have MRF showing lower viscosity in the higher shear rate range. Viscosity 
in the lower shear rate range fairly correlated with MRF stability, i.e., the value of the oil separation ratio decreased as viscosity increased. With regard to stability, the surface characteristics may affect the oil separation ratio results. However, this phenomenon should be considered in light of the interactions among the iron particles, silica particles, and the presence of oleic acid. Finally, the addition of hydrophobic fumed silica can conveniently increase MRF magnetic properties.

\section{References}

Carlson, J. D.; "The Promise of Controllable Fluids," Proc. of Actuator, 94, 266-270 (1994)

Carlson, J. D., D. M. Catanzarite, and K. A. St. Clair; "Commercial Magneto-Rheological Fluid Devices," International Journal of Modern Physics B, 10, 28572865 (1996)

Jolly M. R. and M. Nakano; "Properties and Applications of Commercial Controllable Fluids," Proc. of Actuator, 98, 441-416 (1998)

Kanda, M., A. Kaide, T. Saeki, and H. Tochigi; “A study on the Stability Of Magnetorheological Fluids Prepared with Newly Synthesized Organogelators," Kagaku Kougaku Ronbunshu, 45, 115-122(2019)
Kordonsky, W.; "Magnetorheological Effect as a base of New Devices and Technologies," J. Magn. Magn. Mater., 122, 395-398 (1993)

Lim, S. T., M. S. Cho, I. B. Jang, H. J. Choi, and M. S. Jhon; "Magnetorheology of Carbonyl-Iron Suspensions with Submicron-Sized Filler," IEEE Trans. Magn., 40, 3033-3035 (2004)

Nakano, M., T. Kumasaka, and R. Kudou; "Development of MR Fluid Composite Brake and Its Application Towire Tension Control System for Coil Winding (in Japanese)," Trans. Japan Soc. Mec. Eng., B, 75, 993-999 (2009)

Park, B. J., K. H. Song, and H. J. Choi; "Magneticcarbonyl Iron Nanoparticle Based Magnetorheological Suspension and Its Characteristics," Mater. Lett., 63,1350-1352 (2009)

Rabinow, J. J.; "The Magnetic Fluid Clutch," J. AIEE Trans., 67, 1308-1315 (1948)

Song, K. H., B. J. Park, and H. J. Choi; "Effect of Magnetic Nanoparticle Additive on Characteristics of Magnetrorheological Fluid," IEEE Trans. Magn., 45, 4045-4048 (2009)

Weiss, K. D., J. D. Carlson, and D. A. Nixon; Viscoelastic Properties of Magneto- and ElectroRheological Fluids," J. Intell. Mater. Syst. Struct., 5, 772-775 (1994) 